\title{
Quality evaluation on long stopped building bridges in highway exten- sion projects
}

\author{
H.J.HOU, K.X.XUE, Q.HE, M.L.CUI, J.YAN, R.J.OU \\ College of Civil Engineering and Architecture, East China Institute of Technology, Nanchang, \\ Jiangxi, China.
}

KEYWORD: Highway extension projects; Bridges stopped building for long without maintenance; Quality evaluation; Contrast of quality evaluation criteria; Suggestions for improvement

ABSTRACT: Quality evaluation of bridges stopped building for a long period in highway extension projects is not only critical to re-recognize quality (precondition for follow-up construction) but to adopt treatment measures for bridges. Nevertheless, studies on this issue are quite few at home and abroad so far. In this paper, based on the contrastive analysis of the Chinese bridge technique condition evaluation criteria, the quality evaluation ideas and suggestions are put forward. In the process, it's considered the complexity of bridge engineering, far different from the new, that long stopped now to return to build.

\section{INTRODUCTION}

By the end of 2012, the national highway mileage of 96200 kilometers, first place in the world. Research achievements in the field of construction technology have effectively promoted the development of the industry. But huge highway construction investment scale and insufficient capital investment will cause a sudden stop building, such as the financial crisis of 2008 has led, Shaanxi, Yunnan, Hunan, Jiangxi, Anhui and other provinces multiple highways shutdown for several years. Having sprung up in China more and more BOT projects, and highway extension projects after partial shutdown for long time then continue to build. The related construction market is gradually expanding. However, there are large differences between the highway new projects and extension projects which are more complex than the former.

With the development of the highway quality inspection technology, inspection methods and equipments could meet the detection requirements, under the condition of appropriate combination of them in concrete applications. Related treatment measures could be adopted for bridges that stopped building for a long period now to return to construct in highway extension projects on the basis of correspondingly established subdivision work quality inspection and evaluation. Therefore it is imperative to optimize or to establish new quality evaluation indicators to cope with the problems for bridges that described above.

\section{EXISTING MAIN PROBLEMS}

Highway bridges that stopped construction for long are largely different from new engineering buildings in quality evaluation. Because the quality evaluation mechanism is difficult and complicated, moreover, no established samples can be used for our reference. The difficulty and complexity is the characteristics of the extension projects themselves:

(1) Shutdown time is long, diseases and quality defects of bridges stopped construction for long are significantly greater than the new constructions. This may arouse data missing or lack of credible data about prophase construction, leading the difficulty for quality evaluation according to existing quality inspection evaluation standard; project completion acceptance could not carry out in accordance with the conventional construction projects; no reliable gist for related treatment technology to depend upon, also, we are unable to accurately determine whether the service function of disposed projects meet the requirements or not, and so on.

(2) Unreasonable shutdown interfaces. Artificial and natural factors destruction is more serious along the highway due to stop construction inconsecutively and chronically, leading bridge construction in different interfaces. For example, some pile foundations had been dug but not bored, formworks hanging on pier columns without being taken down and so forth. 
These problems exacerbate the difficulty and complexity of bridge engineering quality evaluation.

\section{contrast of evaluation inspection criteria}

\section{Necessity of the contrast}

At present, the existing three bridge technique condition evaluation criteria in domestic are: the highway bridge standard about maintenance (JTG H11-2004), city bridge maintenance technical specifications (CJJ99-2003) and highway bridge technique condition evaluation criteria (JTG/T H212011). Three in the specification, the highway bridge standard about maintenance has the most widely use scope currently, while the technical specification for city bridge maintenance application is minimal. The implementation time of highway bridge technique condition evaluation criteria is shorter, but it is the most complete specifications so far in bridge technique condition evaluation in domestic.

That the scientific evaluation of current situations of highway bridges which have been built is a prerequisite to decide which kinds of construction need to be repaired, and which kind requires to be rebuilt. To master the present situations of the bridges have been built needs on the premise of analyzing diseases and performances of existing projects. Only a comprehensive understanding of bridge technical conditions to make it accurate investigation of the various performances be possible.

\section{Comparative conclusions}

By comparing we can draw that : the theoretical basis of the assessment methods recommended by the three specifications are the bridge component defect status index method, conventional comprehensive evaluation method, analytic hierarchy process; Bridge quality evaluation inherit the idea of hierarchy analysis generally, and the process of bridge assessment conforms to the process of gradual, from low to high; The long span bridge technical condition evaluation often combine a variety of methods, in order to improve the evaluation precision.

\section{EVOLUTION OF HIGHWAY ENGINEERING QUALITY INSPECTION CRITERIA}

Evaluating pros and cons of the engineering quality is indispensable to every highway construction project work. The effects of quality evaluation: one is to ensure that next procedure could be implemented after the former one has achieved qualification; the other is information feedback. We can accumulate a large number of quality information through the assessment of quality inspection, put forward reasonable quality improvement measures by analyzing what we have collected, so that the engineering quality is in a controlled state and preventing possible problems in the course of construction. Through quality inspection and evaluation, we can not only control the quality of the subdivision work, pick out the unqualified ones to make them meet acceptable quality level after being rectified, but supply qualified products for users.

Highway engineering quality inspection include the engineering quality inspection, engineering quality score and the project quality appraisal and so on, starting from the subdivision work step by step, finally it is concluded that the contract period or construction project quality grades.

As the evaluation unit of highway engineering quality test score, subdivision work quality inspection evaluation contents include four parts: basic requirements, measured projects, appearance appraisal, and quality warranty data. Only cater to the following aspects that quality inspection evaluation of subdivision work could be carried out: materials, semi-finished products and finished products and construction technology that have been used need to meet the requirements of the basic rules, no serious appearance defects, true and fundamentally complete quality warranty information. According to the highway engineering quality inspection evaluation standard (JTJ071-1998), the unqualified item engineering checked by the quality supervision department, is allowed to assess their level of quality again, but can only be reviewed as qualified after the reinforcement, rework, or made to meet the design requirements.

1998 edition of the highway engineering quality inspection evaluation method in determining each test project of component project, using mathematical statistics methods for data processing, and considering all the inspection items of the primary and secondary (weight), basically can objectively reflect the evaluation of the actual situations of the engineering quality. But it is still imperfect:

(1) The test sample is little, which do not conform to the idea of mathematical statistics. In the process of subdivision work test, the number of samples is too little. For example, in compressive 
strength appraisal of concrete, just one group of components at the length less 16 meters and two groups at the length between 16 to 30 meters were selected in upper interior components. However, a small amount of samples does not represent or reflect really the quality of the matrix.

(2) In the process of quality evaluation, the defect of mathematical statistics method is nonnegligible $(\mathrm{Xu}, 2003)$. For example, discreteness of concrete strength is big in construction sites, to determine the qualification rate is not very reasonable obviously with pure mathematical statistics method. Mathematical statistics method is only a means for evaluation of the quality inspection. Although it can provide large amounts of data of quality evaluation for experts, but what kind of ratings should be endowed, pros and cons of the engineering quality, etc. statistical method can do littlie. In quality inspection gauge, the mathematical methods (such as reliability analysis based on the sample estimate, etc.), which are more relevant to the engineering practice, should be adopted as means and methods.

(3) Subjective factors are major problems in appearance identification via expert evaluation. It mainly relates to knowledge level of the individual, authority and different views of engineering structures and so on.

(4) Ambiguity of appearance appraisal score exists, and effectiveness of score deduction is not strong. Highway engineering quality inspection evaluation criteria (JTJ071-1998) gives only a range, which brought difficulty in mastery of the deduction point scale, making the appearance appraisal cannot avoid artificial factors in the actual operation. Especially now that the project quality grades directly associate with the award and punishment of construction unit, which makes the appearance appraisal cannot avoid artificial factors in the actual operation. The direct consequence caused by this malpractice is biased estimation results.

Compared with the 1998 version,2004 edition of the standard have greatly improved, such as increasing the test samples, making the reliability of the test is more in line with the views of mathematical statistics; The construction quality rating only set up a "qualified" and "unqualified", no longer set a "good" rating, etc. But it still has some problems:

(1) Engineering division is not clear. Every project in the construction preparation stage should be divided according to the evaluation criteria come in appendix A. But the regulation on dividing the project in appendix A is too general, thus, some projects in the practical work of division is difficult to determine. For example, foundation and substructure take each bridge or pier and abutment as unit in bridge engineering, however, "prefabrication and installation in superstructure" and "situ casting in superstructure" has not clear division gist, nor the division of piles. In practice, someone divide each pile into two tender subdivision works: the pile foundation and the machining and installing of reinforcing steel bars, while the others take each pile as the subdivision work of the pile foundation and the machining and installing of reinforcing steel bars, which leads to great uncertainty in pile division.

(2) Non-critical parts of itemized project do not stipulate extreme value of deviation (Jiang, 2006). This can result in practical application in a component project according to the evaluation results of criteria may be qualified, but because of deviation of unqualified point of non-critical checked project exceed the permitted range, ultimately affecting the structure and function, and the quality of the next procedure. For example, to assess the highway earthwork roadbed, when longitudinal elevation values of unqualified points are significantly lower than the designed elevation will make the road base excessively thick, leading the density is not easy to meet the requirements after rolling compaction.

(3) Lower limit problem of subdivision works qualified rate.2004 "standard" regulate that, qualified rate of the key project cannot less than $90 \%$, and the detection value shall not exceed the prescribed extreme value, otherwise the process must be reworked. But most of the actual project did not rule the lower limit value of qualified rate, so there may be a checked project qualified rate is low, but the actual project score and level is relatively high. In the example below, we will be able to see the seriousness of the problem: longitudinal elevation qualified rate of a certain rock excavation is zero, while qualified rate of the other subdivision works is $100 \%$, ultimately the project is comprehensively judged as qualified with the rule of weighted scoring system. However, this rock excavation subdivision work is unqualified apparently.

(4) There are some differences in calculation method between highway engineering evaluate standard and highway project completion acceptance: the former take the rate of good unit project, while the latter take construction project evaluation method. 
We can know from the analysis above: although the current 2004 "standard" have greatly improved, but still failed to refine the point deduction standard, weaken subjectivity about exterior appraisal points, improve unclear division about the engineering, did not also deal with lower limit of qualified rate about subdivision work, thus may lead to percent of pass of some inspection items doesn't match with its final rating.

\section{IDEAS AND SUGGESTIONS}

Given to highway bridges that have been stopped construction for a long period to return to construct again, facing the difficulty of the engineering quality evaluation, complexity and no successful examples to refer to, through the analysis above, the author put forward some ideas and suggestions on the basis of reference to the evaluate ideas with considerations of weaknesses for them.

The process of quality evaluation is still follow reasonable application of methods such as the traditional comprehensive evaluation method, the analytic hierarchy process and so on. But we should pay more attention to deal with the following aspects:

(1) Classify bridge diseases first, then to evaluate. Evaluators need to set up evaluation index system, and properly deal with bridges have been built which have "inherent defects", namely waste for tearing down, but even been treated is still unable to make it completely conform to the demand of engineering.

(2) Solve quality evaluation problems under the consideration that lack of credible data about prophase construction. In view of such problems exist universally in bridges long stopped construction then to return to construct, we should not scripted carried out in accordance with the current standards. We can take damage detection and nondestructive testing technology for reasonable evaluation on the quality of the project, of course, allocating the measured item's weight reasonably is necessary.

(3) In view of the "standard", there are no rules for extreme value of deviation about non-critical items in subdivision work inspection, the author recommend evaluators to consult other relevant provisions (such as the municipal road engineering quality inspection evaluation standard (CJJ11990)), and rule that maximum deviation of unqualified points should be within the allowable deviation multiples (such as 1.5 times), or rule allowable deviation maximum value and incorporate it into the specification.

(4) For the lower limit of qualification rate in subdivision works, it is suggested that all kinds of measured projects set rate of the lower limit, such as qualified rate of non-critical projects is not less than $70 \%$, otherwise the unqualified subdivision works.

(5) Qualitative indicator score problems should be handled, in order to solve the operability about the basic requirements check is not strong, the incomplete quality assurance data and problem of human factors on appearance assessment. We can overcome the above problems through the following two approaches: Firstly, perfect the "standard", making the score deducing regime on further refinement; the second is the introduction of fuzzy mathematical method in order to reduce the manmade factors on points, analytic hierarchy process, sequential grading method, etc can be used.

(6) General weights assignment problem in subdivision works. Subdivision works and subprojects can be divided into general engineering project and the main engineering, respectively to a weight of 1 and 2.The operation of grading method is simple, and has the advantage of intuitive thinking clearly. But actual status of bridge engineering, nor the degree of influence for different types of bridges are not identical even the identical subdivision work and subproject. So the above method can't reflect quantitative indicators the size of the contribution to the overall quality of the project truly. It is suggested considering expert's engineering knowledge and his own experience and the authority to give the weight of each component, division of engineering, and at the same time considering the authority of each expert in this field, then to give the weights of experts. The method to determine the weighing values of experts has been mentioned above. Membership function of fuzzy mathematics method can be established to determine the weight of each component, division of engineering.

(7) On fuzziness of engineering division, we can draw lessons from the division method of construction engineering quality test assessment standard (GB50301-2001).

(8) Evaluation criteria and acceptance method should be unified. 


\section{ACKNOWLEDGEMENTS}

First of all, I would like to acknowledge and extend my heartfelt gratitude to my supervisorAssociate Professor Xue Kaixi, for his vital encouragement and patient guidance, generous assistance and invaluable advice, all of which have been of inestimable worth to the completion of my thesis.

Secondly, my special thanks go to all people have helped and taught me in East China Institute of Technology.

Finally, I would also like to thank the support of the National Natural Science Foundation of China (project number: 41462011), College students' innovative training program in 2014 (project number: 2310400148) and Science and technology plan projects in Jiangxi province for 2012 (project number: 20123BBG70214).

\section{REFERENCES}

[1] Jiang Hui. 2006. Highway engineering project quality evaluation system research. Master's thesis. Changsha: central south forestry university of science and technology.

[2] The ministry of communications. 2011. Highway bridge technique condition evaluation criteria (JTG/T H21-2011). Beijing: People's Communication Press.

[3] The ministry of communications. 2004. The highway bridge standard about maintenance (H112004 JTG). Beijing: People's Communication Press.

[4] The ministry of communications. 2004. Highway bridge technique condition evaluation criteria (JTGF80/1-200). Beijing: People's Communication Press.

[5] The ministry of communications. 2003. City bridge maintenance technical specifications (CJJ99-2003). Beijing: People's Communication Press.

[6] The ministry of construction. 2001. Construction engineering quality test assessment standard (GB50301-2001). Beijing: China Architecture \& Building Press.

[7] The ministry of communications. 1998. The highway engineering quality inspection evaluation standard (JTJ071-1998). Beijing: People's Communication Press.

[8] The ministry of construction. 1991. Municipal road engineering quality inspection evaluation standard (CJJ1-1990). Beijing: China Architecture \& Building Press.

[9] Xu Zuen. 2003. Bridge engineering quality evaluation method research. Master's thesis. Xi'an: chang'an university. 